\title{
Erratum
}

\section{Moduli Spaces of Self-Dual Connections over Asymptotically Locally Flat Gravitational Instantons}

\author{
Gábor Etesi ${ }^{1}$, Marcos Jardim ${ }^{2}$
}

1 Department of Geometry, Mathematical Institute, Faculty of Science, Budapest University of Technology and Economics, Egry J. u. 1, H ép., H-1111 Budapest, Hungary. E-mail: etesi@math.bme.hu; etesi@ime.unicamp.br

2 Instituto de Matemática, Estatística e Computação Científica, Universidade Estadual de Campinas, C.P. 6065, 13083-859,

Campinas, SP, Brazil. E-mail: jardim@ime.unicamp.br

Received: 1 October 2008 / Accepted: 20 October 2008

Published online: 28 March 2009 - (C) Springer-Verlag 2009

\section{Commun. Math Phys. 280, 285-313 (2008)}

As it was pointed out by U. Bunke, there is an error in the formulation and proof of Lemma 2.1 in our paper [2]. Hereby we would like to correct it. For sake of clarity we present the whole correctly formulated lemma and its proof.

Lemma 2.1. Fix an $0<\rho<\varepsilon$ and let $\nabla_{A_{\rho}}=\mathrm{d}+A_{\rho}$ and $\nabla_{B_{\rho}}=\mathrm{d}+B_{\rho}$ be two smooth $S U(2)$ connections in a fixed smooth gauge on the trivial $S U(2)$ bundle $\left.E\right|_{\partial M_{\rho}}$. Then there is a constant $c_{1}=c_{1}\left(B_{\rho}\right)>0$, depending on $\rho$ only through $B_{\rho}$, such that

$$
\left|\tau_{\partial M_{\rho}}\left(A_{\rho}\right)-\tau_{\partial M_{\rho}}\left(B_{\rho}\right)\right| \leq c_{1}\left\|A_{\rho}-B_{\rho}\right\|_{L_{1, B_{\rho}}^{2}}\left(\partial M_{\rho}\right)
$$

that is, the Chern-Simons functional is continuous in the $L_{1, B_{\rho}}^{2}$ norm.

Moreover, for each $\rho, \tau_{\partial M_{\rho}}\left(A_{\rho}\right)$ is constant on the path connected components of the character variety $\chi\left(\partial M_{\rho}\right)$.

Proof. The first observation follows from the identity

$$
\begin{aligned}
& \tau_{\partial M_{\rho}}\left(A_{\rho}\right)-\tau_{\partial M_{\rho}}\left(B_{\rho}\right) \\
& =-\frac{1}{8 \pi^{2}} \int_{\partial M_{\rho}} \operatorname{tr}\left(\left(F_{A_{\rho}}+F_{B_{\rho}}\right) \wedge\left(A_{\rho}-B_{\rho}\right)-\frac{1}{3}\left(A_{\rho}-B_{\rho}\right) \wedge\left(A_{\rho}-B_{\rho}\right) \wedge\left(A_{\rho}-B_{\rho}\right)\right),
\end{aligned}
$$

which implies that there is a constant $c_{0}=c_{0}\left(\rho, B_{\rho}\right)$ such that

$$
\left|\tau_{\partial M_{\rho}}\left(A_{\rho}\right)-\tau_{\partial M_{\rho}}\left(B_{\rho}\right)\right| \leq c_{0}\left\|A_{\rho}-B_{\rho}\right\|_{L_{1, B_{\rho}}^{\frac{3}{2}}\left(\partial M_{\rho}\right)}
$$

The online version of the original article can be found under doi:10.1007/s00220-008-0466-9. 
that is, the Chern-Simons functional is continuous in the $L_{1, B_{\rho}}^{\frac{3}{2}}$ norm. A standard application of Hölder's inequality on $\left(\partial M_{\rho},\left.\tilde{g}\right|_{\partial M_{\rho}}\right)$ then yields

$$
\left\|A_{\rho}-B_{\rho}\right\|_{L_{1, B_{\rho}}^{\frac{3}{2}}\left(\partial M_{\rho}\right)} \leq \sqrt{2}\left(\operatorname{Vol}_{\left.\tilde{g}\right|_{\partial M_{\rho}}}\left(\partial M_{\rho}\right)\right)^{\frac{1}{6}}\left\|A_{\rho}-B_{\rho}\right\|_{L_{1, B_{\rho}}^{2}\left(\partial M_{\rho}\right)} .
$$

The metric locally looks like $\left.\tilde{g}\right|_{\partial M_{\rho} \cap U_{\varepsilon}^{*}}=\rho^{2} \varphi\left(\mathrm{d} u^{2}+\mathrm{d} v^{2}\right)+\rho^{4}\left(\mathrm{~d} \tau^{2}+2 h_{\tau, u} \mathrm{~d} \tau \mathrm{d} u+\ldots\right)$ with $\varphi$ and $h_{\tau, u}$, etc. being bounded functions of $(u, v, \rho)$ and $(u, v, \rho, \tau)$ respectively, hence the metric coefficients as well as the volume of $\left(\partial M_{\rho},\left.\tilde{g}\right|_{\partial M_{\rho}}\right)$ are bounded functions of $\rho$, consequently we can suppose that $c_{1}$ does not depend explicitly on $\rho$.

Concerning the second part, assume $\nabla_{A_{\rho}}$ and $\nabla_{B_{\rho}}$ are two smooth, flat connections belonging to the same path connected component of $\chi\left(\partial M_{\rho}\right)$. Then there is a continuous path $\nabla_{A_{\rho}^{t}}$ with $t \in[0,1]$ of flat connections connecting the given flat connections. Out of this we construct a connection $\nabla_{A}$ on $\partial M_{\rho} \times[0,1]$ given by $A:=A_{\rho}^{t}+0 \cdot \mathrm{d} t$. Clearly, this connection is flat, i.e., $F_{A}=0$. The Chern-Simons theorem [1] implies that

$$
\tau_{\partial M_{\rho}}\left(A_{\rho}\right)-\tau_{\partial M_{\rho}}\left(B_{\rho}\right)=-\frac{1}{8 \pi^{2}} \int_{\partial M_{\rho} \times[0,1]} \operatorname{tr}\left(F_{A} \wedge F_{A}\right)=0,
$$

concluding the proof.

This lemma is used in the estimates on p. 293 and p. 299 in [2]. In these estimates, the original (incorrect) $L^{2}$ norm of the $\mathfrak{s u}(2)$ valued 1 -form $A_{\rho}-\Gamma_{\rho}$ should be replaced simply by its $L_{1, \Gamma_{\rho}}^{2}$ norm dictated by the corrected Lemma 2.1 presented here. This replacement is only of technical nature and does not effect any of the main results in [2].

Finally, we would like to thank U. Bunke for pointing out this technical gap.

\section{References}

1. Chern, S., Simons, J.: Characteristic forms and geometric invariants. Ann. Math. 99, 48-69 (1974)

2. Etesi, G., Jardim, M.: Moduli spaces of self-dual connections over asymptotically locally flat gravitational instantons. Commun. Math. Phys. 280, 285-313 (2008) 